\title{
PATHOGENICITY OF FUNGI AND BIOASSAY DESIGN
}

\author{
RICHARD A. HALL
}

\author{
70, Parkside Avenue, Littlehampton, West Sussex BN17 6BJ, England
}

\section{WHAT IS BIOASSAY?}

Bioassay is the quantification of a biological parameter. Here, we are considering the pathogenicity of fungi which attack insects. What we choose to measure depends upon the objectives and it is surprising how many scientists simply copy the efforts of others without giving due regard to what they are trying to achieve. For example, if the aim is to investigate the fundamental mechanisms goveming pathogenicity in a fungus, e. g. studying the relationship between the expression of say an exo-enzyme and ability to infect, then a simple assay system is sufficient to determine the percentage mortality in a laboratory insect population treated with fungus. However, if the requirement is to select or check a fungus for its ability to control insects in the field, then some refinements are required; the ideal bioassay is an experiment in the field. Of course, when comparing many strains or when doing routine quality control, this is totally impractical. So a meaningful bioassay system representing a microcosm of the field situation should be designed and attention must be paid to the physical conditions, e. g. temperature and humidity, which prevail there. In addition, one must consider the nature of the insect pest and the epizootiology of the pathogen. For example, one cannot always assume that a fungus possessing a good infectivity in the laboratory for a standardised insect population will necessarily spread well amongst a rapidly reproducing insect population in the field.

Thus, there are at least two important parameters which need to be considered when designing a bioassay system: infectivity, that is simply the measurement of the ability of a fungus to induce mortality in insects treated with infectious material, and epizootic potential, a measurement or representation of which would provide an idea of the ability of a fungal pathogen to spread within a field insect population following introduction of the disease into the population in the form of e. g. a spore spray.
The following discussion will highlight these factors and stress others which must be considered in order that a meaningful bioassay system can be designed.

\section{BIOASSAY OF INFECTIVITY}

Dose Response Curve - In any kind of bioassay, one is looking for some sort of response at one or more doses. In the present context, this is usually mortality which increases with increasing spore dosage. If we simply plot mortality by dosage, we get a sigmoid curve. This is normally transformed to a mathematically more easily managed straight line by converting dosage to $\log _{10}$ dosage and mortality to probits (Finney, 1978). The data are computer analysed to obtain the best estimate of the $\mathrm{LC}_{50}$ or the dosage at which $50 \%$ of test insects are killed. There are very few systems which have been developed which actually measure a known dose of fungal spores to insects leading to an estimation of an $\mathrm{LD}_{50}$. Even so, where spore concentrations are used, the $\mathrm{LD}_{s 0}$ can still be approximated by washing insects treated with an $\mathrm{LC}_{50}$ and counting colonies arising on agar plates a few days later.

For a complete treatise on how to analyse bioassay data consult Finney (1978).

$\mathrm{LT}_{50 \text { s }}$ (the time taken to kill $50 \%$ of an insect population) are not as sensitive as $\mathbf{L C}_{50}$ determinations because observations tend to be taken only every $24 \mathrm{~h}$. Nevertheless, since just one dosage is normally used, $\mathrm{LT}_{50}$ assays require relatively little labour input. The concentration selected must not be too high otherwise any true differences in pathogenicity may be obscured.

Effect of Quality of Insects on the Accuracy of Bioassay - The standard error of the $\mathrm{LC}_{50}$ depends largely on the value of the slope of the dosage mortality line which in turn depends in part on the heterogeneity of the test insect 
population; some insects may be very sensitive to the fungus while others may be comparatively resistant. Such a scattered response means that the slope of the dosage - mortality curve will be low. Quite simply, the lower the slope, the less accuracy can be assigned to the estimate of the $\mathrm{LC}_{50}$. If the slope is too low, then a significant $\mathrm{CHI}^{2}$ result will mean that the slope is essentially not different from zero which really means there is no dosage - mortality relationship. To achieve maximum homogeneity, insects should be reared under conditions held as constant as possible (temperature, relative humidity, light) and the quality of diet should likewise not change, though seasonal influences are difficult to avoid. A further refinement aimed at making slopes as steep as possible is to employ a selection technique to remove the weaker and more susceptible insects from a population; for example vigorous insects tend to be more mobile and agile and, particularly if they can be attracted to or, repelled by stimuli, may be separated from the weaker members of a population.

\section{Quality of the Fungal Inoculum; Standard} Isolate; Potency Ratios - Spores, either produced on solid substrate (agar, bran, etc.) or in liquid fermentation are usually the only infective propagules used in bioassay. To obtain reproducible results in consecutive bioassays, spores should be produced under constant conditions on a substrate of consistent quality. Also, spores should not be allowed to deteriorate and lose viability or infective vigour prior to being used for assay; fungal spore pathogenicity can decline disproportionately faster compared to spore viability (Hall, unpublished data). Therefore, the target spore viability should always be neas $100 \%$. Viability of spores is easily checked by an overmight germination test on an agar slide (Hall, 1976).

To further reduce variation between assays, it is suggested that identical aliquots of spores derived from the same culture of the same single spore isolate be preserved in the deepfreeze or preferably in liquid nitrogen and used to culture up spores for bioassay when required. Such a single spore isolate would be designated as a standard to be used in each consecutive assay. By obtaining the ratio:

$$
\frac{\mathrm{LC}_{50} \text { test fungus }}{\mathrm{LC}_{50} \text { standard }}
$$

a good deal of the inevitable variation arising between consecutive assays can be eliminated. In parenthesis, the researcher should satisfy himself that his single spore isolate is representative of the fungal spore population in his standard strain. Single spore isolates of Verticillium lecanii exhibited identical pathogenicity (Hall, 1980) but this was not so for at least one strain of Beauveria bassiana (Hall, unpublished data).

Little need be said about the type of propagule used for bioassay. As bioinsecticides, blastospores (produced in liquid fermentation) are normally the inoculum of choice since they can be more rapidly and cheaply produced on an industrial scale than conidia though some fungi e. g. Metarhizium anisopliae do not produce blastospores so readily in liquid media. Blastospores are often slightly more pathogenic than conidia (Hall, 1979) and, contrary to popular opinion are not difficult to stabilize and formulate.

Mode of Application of Infectious Material - Unless other specific effects are being studied, the mode of application of infectious material should be similar to that used in the field. This, however, is often difficult to reproduce in a precise manner in the laboratory so a compromise has to be found. The most frequent method of infecting insects is by brief immersion in spore suspensions, or for aquatic insects, holding them in known aqueous spore concentrations. Other workers (Ignoffo et al., 1976) coat leaf discs with spores which are ingested by the (lepidopterous) insect host. Apart from the oral route, acquisition of disease from leaf surfaces by insects tends to be inefficient and variable (Hall, 1977, unpublished data). Infection of certain insects - particularly those in soil - can be slow and a meaningful bioassay system may require many weeks. In the case of Vine weevil (Otiorrynchus sulcatus) this problem can be overcome by infecting the more susceptible early developmental stages on moistened filter paper; such an assay can be read in a few days as opposed to weeks if a soil inoculation system is used.

Wetting agents can be a mixed blessing; they are certainly necessary to wet very hydrophobic cuticles of some insect species but I have found that aphids which were thoroughly wetted by Triton X-100 or Tween 80 were no more efficiently infected than comparable aphids 
immersed in spores in pure distilled water (and which were certainly not visibly well wetted). Because wetting agents may exert some serious and unexpected negative in vivo effects such as impairment of sporulation on the cadaver (Hall, 1982), one should take care to select a wetting agent which is truly innocuous. They are certainly use ful for dispersing clumps of spores particularly the very dry conidia of Metarhizium anisopliae. Even so, prolonged agitation in distilled water of even Metarhizium spores will eventually result in dispersion. The message is, in bioassay if you do not really need a wetting agent, don't use it and this applies to the field situation also.

Environmental Conditions of Bioassay What temperature and humidity conditions does one select for bioassay? If the emphasis is on rapid results as for when one is attempting to correlate a character (e. g. an exo-enzyme) with pathogenicity, or for routine quality control in a production system, there is no reason why the assay should not be carried out at continuous high $\mathrm{RH}$ and at the temperature optimum for the growth of the fungus. If, on the other hand, one is screening for a strain of "fungus which will do the best job in the field, then the temperature which prevails during the period when infection is likely to take place i. e. diurnally high RH, should be selected. Ideally, the RH should be alternated diurnally to mimic outdoor conditions but this is a difficult and expensive refinement to introduce and here growth cabinets are the ideal solution. However, using an appropriate field temperature is a start and this will enable the researcher to identify promising candidate fungi.

Concentration of Spore Dosage and Duration of Assay - If very high spore dosages are used, insects can be killed remarkably quickly due to blocking of spiracles or massive disruption of the cuticle caused by wholesale invasion. This certainly occurs with $V$. lecanii which, at a concentration of $5 \times 10^{8}$ spores $/ \mathrm{ml}$ kills aphids in $24 \mathrm{~h}$. But such high doses are of academic interest only. Economical concentrations of spores are in the range $10^{5}$ to $10^{6}$ spores $/ \mathrm{ml}$ and it is approximately this range which is used for bioassaying this fungus. Assays with $V$. lecanii last six days (Hall, 1976). By this time, an $\mathrm{LC}_{50}$ has been obtained and control mortalities are still low. Beyond six days, control mortalities increase considerably while the $\mathrm{LC}_{50}$ changes comparatively little.
Therefore the duration of an assay system should be sufficiently long to permit the $\mathrm{LC}_{50}$ to reach a plateau at practical spore concentrations but not be so long that excessive control mortalities occur. This can be achieved for most species of insects but some such as the notorious Brown planthopper of rice (Nilaparvata lugens) will exhibit a high control mortality no matter how careful one is; only by minimal handling and disturbance of these insects can the control mortality be kept at 10 to $15 \%$ (after 5 days) compared with about $0 \%$ for most other insects. Much thought and effort should be given to reducing control mortality since this contributes to the accuracy and reproducibility of the assay system.

\section{MEASUREMENT OF EPIZOOTIC POTENTIAL}

When fungi are used to control certain insect pests, particularly those with a high reproduction potential, application of spores onto a crop serves only to introduce disease into the insect population. The spores in the spray alone cannot possibly control for example aphids or whiteflies (Hall \& Papierok, 1982) but they establish foci of infection from which disease spreads to healthy insects. Many strains of $V$. lecanii exhibit similar infectivity in laboratory bioassay but differ enormously in their abilities to achieve control in a population of rapidly reproducing insects (Hall, 1984).

The ability of a disease to spread depends not only upon a strong inherent pathogenicity or infectivity but also on other factors such as speed of infection, speed and extent of sporulation on the insect cadaver. The mode of sporulation is also important, e. g. spores of some strains of $V$. lecanii may be shrouded on the cadaver by sterile mycelium leading to a reduced epizootic potential (Hall, 1984).

Obviously, it would be laborious in the extreme to measure each of the parameters which impinge upon epizootic potential when screening large numbers of strains or species. In the case of $V$. lecanii, the problem was solved by simply measuring secondary mortality in laboratory aphid populations in the following way. A suitable spore concertration was selected as a result of experience with a conventional assay system (Hall, 1976) and a concentration which also reflected the economical field dosage. Adult aphids are immersed briefly in spore suspensions (ca. $10^{6}$ 
spores $/ \mathrm{ml}$ ) of the standard and test strains of $V$. lecanii. At the end of the six day assay period at $20^{\circ} \mathrm{C}$ mortality approaches $100 \%$ and cadavers are covered in sporulating mycelium. The epizootic potential of the different strains is compared by determining the proportion of dead progeny aphids born to the adults during the six day period. The Table shows unequivocably that while strains may elicit the same \% mortality amongst spore treated adults, the subsequent spread of disease to progeny varies greatly. Those strains which kill relatively few progeny in this kind of bioassay perform very poorly in the field (Hall, unpublished data).

TABLE

Adult and progeny aphid mortalities resulting from treatment of adults with spores of different V. lecanii strains

\begin{tabular}{rrr}
\hline Strain & $\begin{array}{c}\text { Adult Mortality } \\
\%\end{array}$ & $\begin{array}{c}\text { Progeny Mortality } \\
\%\end{array}$ \\
\hline $1-72$ & 100 & 84 \\
$57-81$ & 91 & 9 \\
$79-82$ & 100 & 0 \\
$18-78$ & 91 & 8 \\
\hline
\end{tabular}

In other assay systems, attempts to quantify epizootic potential throw light on the true ability of fungal pathogens to control pests. Metarhizium anisopliae is strongly pathogenic in terms of infectivity to Brown planthopper of rice but secondary infection studies demonstrated how poor a control agent it really is against this pest (Hall, unpublished data).

\section{CONCLUSION}

To conclude, it is evident that in designing a bioassay system, all factors conceming the characteristics of, and the relationship between the fungal pathogen and the target insect host, as well as the objectives of the planned investigation, must be considered very carefully. Experimenters must select appropriate parameters which take into account the practical difficulties that an ideal bioassay system would engender. Where objectives are highly practically-orientated, the assay should be designed with particular attention being paid to the usual conditions of temperature and humidity which prevail in the field. Where post -applicator spread of disease is essential to control a pest population, methods of comparing epizootic potential should be developed; often it is simply not good enough to determine just the $\mathrm{LC}_{50}$ or a similar parameter.

\section{REFERENCES}

FINNEY, D. J., 1978. Probit Analysis. Cambridge University Press, Cambridge.

HALL, R. A., 1976. A bioassay of the pathogenicity of Verticillium lecanii conidiospores on the aphid Macrosiphoniella sanborni. J. Invertebr. Pathol., 27: $41-48$.

HALL, R. A., 1977. The Potential of the fungus Verticillium lecanii as a microbial control agent of glasshouse pests. Ph. D. thesis, University of Southampton.

HALL, R. A., 1979. Comparison of the pathogenicity of Verticillium lecanii conidiospores and blastospores against aphids. Entomophaga, 24: 191-198.

HALL, R. A. \& BURGES, H. D., 1979. Control of aphids in glasshouses with the fungus. Verticillium lecanii. Ann. Appl. Biol., 93: 235-246.

HALL, R. A., 1980. Effect of repeated subculturing on agar and passaging through an insect host on pathogenicity, morphology and growth rate of Verticillium lecanii. J. Invertebr. Pathol., 36: 216-222.

HALL, R. A., 1984. Epizootic potential for aphids of different isolates of the fungos, Verticillum lecanii. Entomophaga, 29: 311-321.

HALL, R. A. \& PAPIEROK, B., 1982. Fungi as biological control agents of arthropods of agricultural and medical importance. Parasitology, 84: 205-240.

IGNOFFO, C. M.; PUTTLER, B.; HOSTETTER, D. L. \& DICKERSON, W. A., 1976. J. Invertebr. Pathol., 28: 259-262. 